\title{
Interactive comment on "Improving GOES Advanced Baseline Imager (ABI) Aerosol Optical Depth (AOD) Retrievals using an Empirical Bias Correction Algorithm" by Hai Zhang et al.
}

\section{Anonymous Referee \#1}

Received and published: 15 April 2020

The goal of this paper is to correct for AOD retrieval biases in GOES ABI AOD product using an empirical approach.

The surface reflectance in the current GOES AOD algorithm is estimated based the relationship between 0.47 and $2.2 \mathrm{um}$ and 0.64 and 2.2 um since most aerosols are 'transparent' in the 2.2 um. This is based on the Kaufman et al (1997, IEEE) paper that many MODIS algorithms use to estimate surface reflectance. In this paper, the authors look for 'clear days' (based on AERONET AOD values less than 0.05) to assess the GOES AOD (for both high and medium quality retrievals) for a few selected sites. They report that the GOES AOD is biased since the GOES AOD is much larger than the 
AERONET AOD for these clear days. The authors note that the biases appear to be centered around 1700 UTC and it is due to surface reflectance parametrizations at various sun-satellite viewing geometries. The authors then attempt to correct this bias based on the premise that it is the surface reflectance that is the issue in the GOES algorithm. Then they use a 30-day composite of GOES AOD to estimate the minimum $A O D$ and subtract that with the background AOD (a fixed value of .025) to correct for the bias. They use two polynomial fitted relationships to estimate biases. They then correct the AOD using these relationships and then validate the results with AERONET AOD and show improvement in these biases.

First I need to note that the paper needs to go through some editorial clean up since several sentences are awkward; key references (Kondragunta et al 2020) are missing; and some references are really old.

I find several problems with the paper and most importantly it is the use of AOD to make these corrections rather than working with the reflectances. The algorithm retrieves AOD based on apriori assumptions of aerosol model, surface parametrizations based on NDVI , cloud clearing approaches and a host of thresholds for cloud cover, inhomogeneity, etc (ATBD, 2018). Now this paper indicates that the surface parametrizations are a problem and then to remove the biases the authors use the retrieved AOD to make bias adjustments. The original algorithm uses reflectance ratios to arrive at surface values and now this paper goes back to the older GASP approach to obtain the 30-day composite minimum (not reflectance) AOD values. Looking at this from an algorithm perspective it is not the correct solution for an operational algorithm to go through retrieval using one set of processes, retrieve AOD's and then use the retrieved AOD values to make corrections for parameters that are part of the original retrieval process (in this case surface reflectance). The authors need to think about having the correct algorithm as part of the retrieval process rather than adjusting it after the retrieval is done.

The other issue is the relaxation of quality flags to allow more data. There were strong 
reasons for picking all the metrics for high and medium quality flags in the first place (ATBD, 2018) whether it is cloud/snow cover or inhomogeneity. Line 90 to 95 provides the various reasons for selecting the pixels for the retrievals and this paper now allows all the medium quality flags in the process but does not address cloud contamination issues. The paper needs to be more convincing that it is indeed surface issues and not cloud cover that causes these problems. The results need to be discussed in terms of scattering angles (see She et al, Remote Sensing, 2019). This will allow more quantitative analysis rather than statements like those in 160-161. Also for Figure 1 and Figure 2 what were the histograms of actual reflectance's from the GOES channels for the various peaks. This can help explain Figure 2 better.

The paper uses two sets of parametrizations for adjusting the biases and then in line 255 back tracks the approach by stating that this could have large uncertainties.

Figure 8 and Figure 9 appears as a complete afterthought since the aerosol model discussion is not complete or convincing. I have no idea why the PM2.5 discussions (Figure 9) is relevant for this paper.

The AERONET data used is from 2018 and the authors need to be using Level 2 not 1.5. This data should be available.

Other issues. Define accuracy and precision and be quantitative rather than merely stating that one product is better than the other. Line 50, Deemed to have quality sufficient is rather vague. Line 73: The word transparent to most aerosols is rather vague. Describe why this is possible briefly based on aerosol size and extinction Line 74. Again, poor phrasing. It is not linear reflectance BETWEEN channels if it is three channels. Be specific. Line 75-80 is awkward phrasing. The algorithm does not make retrievals? Describe the algorithm clearly but briefly. Line 81-84 is not clear at all. While I understand how the algorithm works this type of writing will not help all readers understand the algorithm and methods used in this paper. Line 89: Usually very small? What does that mean? Need some numbers. Lines 89-94 needs to be clearer with 
brief discussion rather than listing the problems. Line 98: If the surface reflectance issues are so different between 0.41 and 0.47 micron then the authors need to show or discuss this for certain land types. Otherwise these statements are vague. 115-120 discussion is not "technical" enough. What does air mass movements mean? You need to then state what wind speeds at what height provide the $27.5 \mathrm{~km}$ radius. Line $140+$ : How about retrieval biases due to sun-satellite viewing geometry in radiative transfer code? Line 147: We need to see these relationships between two channels for the solar geometries.

I find the two reasons in 152-155 to be problematic. Why should the test position issue matter if these relationships are established for certain solar viewing geometries/NDVI? Plus there are reasons why the quality flags were established for high, low, medium in the first place (cloud cover, snow cover etc). Of course one would use the best quality flags for establishing surface reflectance relationships because of contamination issues. Now if you are using medium quality flags to get more data into the analysis then of course your surface reflectance relationships are going to be different.

Since this paper is about surface reflectance issues the authors need to show these relationships that currently exist for various angles/NDVI first to make their case stronger.

The authors should also show the reflectance values on these plots so we can interpret the results better.

Interactive comment on Atmos. Meas. Tech. Discuss., doi:10.5194/amt-2020-115, 2020. 